\section{IMMEDIATE BIOLOGICAL BENEFITS}

MIS Implants introduces the radically new V3 dental implant for immediate biological benefits.

The unique triangular shape of the coronal portion of the new V3 Implant enables a significant gain of bone and soft tissue volume where it matters most so less titanium and more bone and soft tissue are available.

The triangular neck of the V3 provides solid anchorage at three points in the crestal zone while forming three compression-free gaps at the sides: between the implant and the osteotomy. This design is aimed at high primary stability and the reduction of bone compression and crestal bone resorption: favoured conditions for increased osseointegration.

The new V3 Implant is the result of an intensive two-year R\&D process by MIS, along with their development partners Professor Nitzan Bichacho, Dr Eric VanDooren and Dr Yuval Jacoby. The result is a high performance conical-connection implant with platform switching, a variable thread and self-tapping capability, micro-rings, a concave interthread for maximum BIC, and a flat apex supporting immediate placement engagement.

The new V3 design aims to provide surgical specialists and GPs alike with optimum flexibility in implant planning and placement for a restorative-driven approach.

For more information visit www.mis-implants.com.

\section{FLOWABLE AND YET PACKABLE}

Particularly fast, particularly natural: IonoStar Plus from VOC0, the newly developed glass ionomer restorative material in the innovative application capsule, is ideal in cases requiring a fast and aesthetic solution. It has four significant advantages over other glass ionomers: very easy to extract from the capsule, the material at first has excellent wetting characteristics, before changing its viscosity and then being malleable for at least one minute without sticking. Flowable, yet packable - this makes IonoStar Plus the only glass ionomer material to provide ideal viscosity at every stage of application.

Moreover, the material is notable for its speed. After only 10 seconds of mixing it is soft, and within three minutes of starting application it is ready for smoothing. The curing time is just

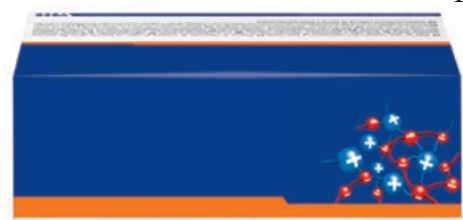
two minutes. This is a valuable advantage, particularly in the treatment of patients with low compliance, IonoStar Plus

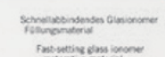
such as children.

IonoStar Plus is also the very first GI cement to offer fluo- rescence corresponding to the natural tooth, so there is no aesthetic impact even when treating anterior teeth, for example in the case of class V fillings. Its particularly high fluoride release counteracts the formation of secondary caries.

IonoStar Plus is available in the new VOCO application capsule, which can be used without an activator. It is simply pushed down onto a firm surface (such as a table) so that the coloured plunger is pushed inside the capsule. Then it is mixed as usual in a highfrequency mixer for 10 seconds. After insertion into a standard applicator, the application cannula of the capsule just needs to be turned upwards and the material is ready for filling. The new capsule design also makes it easier to reach smaller cavities and difficult-toaccess areas in the mouth.

For further information contact 07500769613.

\section{OBJECTIVE IMPLANT STABILITY}

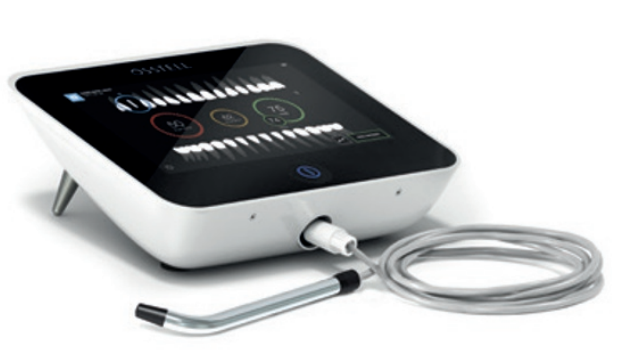

Osstell has launched the Osstell IDx - a completely new and improved platform with a novel, intuitive user interface and functional design. The Osstell IDx is combined with the new Osstell Connect - a set of online services for automatic ISQ data storage, sharing and analysis of implant stability data.

Osstell now takes the next leap in the evolution of implant diagnostics with the introduction of Osstell IDx. It displays the ISQ measurements in an intuitive and easily interpreted way. It is easier than ever to assess implant stability and the degree of osseointegration, ensuring that implants are stable enough for final restoration. It also facilitates communication with patients, or restorative dentists, concerning the treatment plans.

Furthermore, with the Osstell IDx the company also introduces the associated online service Osstell Connect. Implant stability data and results are automatically stored directly in the device and in the Osstell Connect service. It makes collaborations with colleagues easier, enables data backup, remote service and support, and allows the user to analyse implant and treatment data through various platforms.

For further information visit www.osstell.com

\title{
TAKE HEED OF RESTRICTIVE COVENANTS
}

The property experts at Goodman Grant Solicitors understand that practice owners and principles should be aware of restrictive covenants when undertaking property purchases, expansions or a change of use.
Restrictive covenants are frequently overlooked. But, if there is a covenant on your lease that restricts or controls your activities, you could run into costly problems if you are found to be in breach.

Goodman Grant Solicitors is one of the country's foremost law firms providing specialist legal services to the dental profession, If you are looking to buy, sell or lease a dental practice and want to avoid nasty surprises due to restrictive covenants, seek out the expert guidance and advice of the specialists at Goodman Grant.

For more information call 0151 7070090 oremail ik@goodmangrant.co.uk. 